Jérôme Jacquin

\title{
Le/La polémique : une catégorie opératoire pour une analyse discursive et interactionnelle des débats publics?
}

\begin{abstract}
Avertissement
Le contenu de ce site relève de la législation française sur la propriété intellectuelle et est la propriété exclusive de l'éditeur.

Les œuvres figurant sur ce site peuvent être consultées et reproduites sur un support papier ou numérique sous réserve qu'elles soient strictement réservées à un usage soit personnel, soit scientifique ou pédagogique excluant toute exploitation commerciale. La reproduction devra obligatoirement mentionner l'éditeur, le nom de la revue, l'auteur et la référence du document.

Toute autre reproduction est interdite sauf accord préalable de l'éditeur, en dehors des cas prévus par la législation en vigueur en France.
\end{abstract}

revues.org

Revues.org est un portail de revues en sciences humaines et sociales développé par le Cléo, Centre pour l'édition électronique ouverte (CNRS, EHESS, UP, UAPV).

Référence électronique

Jérôme Jacquin, « Le/La polémique : une catégorie opératoire pour une analyse discursive et interactionnelle des débats publics ? », Semen [En ligne], 31 | 2011, mis en ligne le 01 avril 2011. URL : http://semen.revues.org/ pdf9085

DOI : en cours d'attribution

Éditeur : Presses universitaires de Franche-Comté

http://semen.revues.org

http://www.revues.org

Document accessible en ligne sur : http://semen.revues.org/pdf/9085

Ce document PDF a été généré par la revue.

Tous droits réservés 
Jérôme JACQUIN

CLSL (Centre de linguistique et des sciences du langage)

Université de Lausanne

\section{Le/La polémique : \\ une catégorie opératoire pour une analyse discursive et interactionnelle des débats publics ?}

La présente étude s'interroge sur le caractère opératoire de la catégorie «polémique » dans un contexte de débat public. Y a-t-il lieu d'y repérer des moments "polémiques»? La question se pose avec plus d'acuité encore lorsque le mot lui-même est absent du corpus ${ }^{1}$, c'est-à-dire lorsqu'aucun locuteur ne catégorise par ce terme le comportement verbal d'un ou des participant(s) en présence. Si l'analyste a néanmoins le sentiment qu'en certains endroits de son corpus, le régime change de manière reconnaissable, et qu'une certaine polémicité affleure à la surface du débat, à partir de quels critères va-t-il vérifier l'hypothèse de l'émergence d'un segment «polémique»? De fait, le caractère opératoire de la catégorie «polémique» pour l'analyse de débats publics semble dépendre de l'articulation que l'on propose entre un point de vue savant et le point de vue des participants, entre l'intuition que " quelque chose de spécifique se passe » et l'analyse du comportement discursif et interactionnel des participants à l'interaction en tant qu'orienté vers ce "quelque chose». Notre question générale de recherche sera ainsi la suivante : «En quoi peuton relever des traces d'orientation des participants vers la réalisation d'une forme (discursive ou interactionnelle) dite polémique ?

Pour éprouver le caractère opératoire de la catégorie, je soumets deux thèses à la discussion. La première consiste à dire que l'étude du phénomène polémique n'exige pas seulement un repérage systématique de formes caractéristiques, mais nécessite également la prise en compte des évaluations produites par les acteurs : le phénomène polémique - comme tout type d'action - est une forme certes, mais qui fait l'objet d'une évaluation en contexte. Complémentaire à la thèse de l'évaluation contextuelle, la seconde thèse défend, dans la lignée des travaux de KerbratOrecchioni (1980), l'introduction systématique d'une distinction entre «le » polémique (en tant que forme communicative ou « action verbale » produite

1. Le corpus de cette recherche doctorale est composé de huit événements interactionnels de type «débat public ». Ces événements ont été enregistrés (une dizaine d'heures au total) sur support audio/vidéo, puis transcrits. 
par un locuteur) et «la» polémique (en tant que type d'échange ou «pattern interactionnel» dans lequel s'engagent des locuteurs) ${ }^{2}$. La contribution entend montrer que la prise en compte de l'évaluation permet de penser le passage du régime discursif au régime interactionnel du phénomène polémique.

Après avoir introduit la spécificité du débat public comme pratique sociale (1), je présente brièvement le cadre conceptuel permettant d'interroger la catégorie " polémique » (2), ainsi que les considérations des analystes de discours et des interactions s'étant penchés sur le phénomène (3). J'analyse ensuite mon corpus afin d'éprouver les traits définitoires dégagés (4). Le retour sur les différents extraits analysés est l'occasion de souligner la présence systématique d'évaluations produites par les acteurs engagés dans le moment "polémique»: je défendrai que la nature polémique ou non d'une activité verbale (un discours ou un moment d'interaction) dépend avant tout des «jugements de polémicité » des participants (5). Enfin, la présence des évaluations me permet également de penser la possibilité d'une articulation entre le régime discursif «du» polémique et la dynamique interactionnelle «de la» polémique. La contribution se termine par une réflexion sur l'importance de considérer l'évaluation comme catégorie sociohistorique : si les participants jugent passer de l'argumentatif au polémique et du débat à la polémique, ils le font à l'aune de normes socialement et historiquement déterminées distinguant le licite et l'illicite.

\section{Le débat public : un lieu spécifique d'expression politique}

Je m'intéresse aux débats politiques en tant qu'ils prennent place dans des situations particulières de communication publique qui m'autorisent à parler par la suite de «débats publics». De tels événements sont identifiables par :

a) la co-présence spatiale et temporelle de l'ensemble des participants, public compris. Les débats publics, vidéo-enregistrés par une équipe de recherche, impliquent une incarnation radicale de la parole politique, un dispositif proxémique spécifique (c.-à-d. une répartition géospatiale particulière des participants) informant la dynamique interactionnelle et l'exploitation par les participants de ressources tant verbales que mimogestuelles. Au plan méthodologique, la clôture spatiale de ces événements filmés dans des salles de cours de l'Université de Lausanne - favorise pour le chercheur une prise de vue relativement englobante de leur écologie

2. Si cette distinction fera l'objet d'un commentaire spécifique, elle est néanmoins utilisée de manière systématique tout au long de la contribution. 
(c. à d. leur développement dans le temps et l'espace). Les débats publics se caractérisent également par leur caractère éphémère : exception faite de l'enregistrement réalisé par l'analyste, aucune fixation sur support imprimé ou numérique n'est effectuée.

b) le caractère politique des thématiques et enjeux abordés (p. ex. le financement des études supérieures), des catégorisations opérées sur les participants (p. ex. des candidats aux élections, des membres de partis, des responsables d'initiatives ou de référendum ${ }^{3}$ ) et des contextes de pertinence invoqués (p. ex. un contexte électoral).

c) leur organisation par des institutions associatives non professionnelles (et non lucratives). Il en va de même pour les invités qui, quand bien même ils sont le plus souvent au bénéfice d'un mandat politique (député, président de section régionale de parti, responsable d'une initiative ou d'un référendum), n'en sont pas pour autant des professionnels de la politique ${ }^{4}$. Compte tenu des points (a) et (b), les débats publics témoignent d'un mode particulier d'expression de la parole politique : une politique de proximité, une politique de terrain valorisant le dialogue entre citoyens et personnel politique.

d) Leur médiatisation (sommaire, mais bien réelle). Aussi minimales que soient les ressources dont ils disposent (courriers électroniques envoyés via les mailing-lists de l'administration ; affiches placardées sur les murs de l'université ; annonces orales en contexte formel ou informel), les organisateurs des débats publics jouent un rôle fondamental de médiateur. Si les rencontres ont pour but de s'affranchir de cadres institutionnels et médiatiques trop contraignants en favorisant un dialogue direct entre les instances de la vie politique locale, il n'en reste pas moins que leurs organisateurs ont pour tâche de rendre les rencontres publiques, d'en faire des événements auxquels tout un chacun est invité à assister et participer.

e) un dispositif général favorisant la distinction et l'agonicité entre intervenants. Les débats publics visent à rassembler des voix contradictoires. Pour ce faire, la thématique est le plus souvent annoncée par une question fermée ( $\mathrm{p}$. ex. «faut-il interdire les véhicules trop polluants ?»). Suivant les modèles de Christian Plantin (2005), de Marcel Burger (2005) ou encore de Ian Hutchby (1996), l'activité de débat

3. Le lecteur intéressé par le fonctionnement de la démocratie helvétique trouvera toutes les informations utiles sur le site internet de l'administration fédérale :

http://www.bk.admin.ch/themen/pore/index.html?lang=fr

4. D'après les analyses de Heidi Z'graggen et Wolf Linder (2004), la politique suisse est l'une des moins professionnalisées d'Europe. Loin du personnel politique français (contexte peut-être plus familier au lecteur francophone), la Suisse se distingue par la semi-professionnalisation de son personnel politique, celui-ci cumulant le plus souvent un emploi et un mandat ou engagement politique. 
s'organise autour de la confrontation entre propositions émanant d'acteurs différents et polarisés par le biais de catégorisations identitaires (p. ex. « libéral », " conservateur », « socialiste »).

Cette recherche tire son originalité de l'enregistrement et de la description de formes ordinaires, locales et relativement artisanales - très éloignées des phénomènes de médiatisations de masse - de communication et d'interaction à caractère politiques ${ }^{5}$. Événements de confrontation in praesentia, confrontation d'opinions portées par des êtres de chair et de sang, les débats publics constituent autant de lieux pertinents pour interroger la catégorie "polémique» en tant qu' «usage social de la parole $»^{6}$ engageant radicalement les personnes, leur image, leurs arguments respectifs.

\section{Quelques mots sur l'interactionnisme socio-discursif}

Mon propos s'inscrit dans le cadre général de l'interactionnisme sociodiscursif (voir Bronckart 1997 ; Filliettaz 2002). Hérité d'une constellation de recherches ${ }^{7}$ autour des propriétés de l'interaction, ce paradigme insiste sur plusieurs points :

a) Le caractère typifié des pratiques. L'idée générale consiste à dire que les pratiques sociales impliquent un double mouvement de typification, attaché tant à la production qu'à la réception des actions : tandis que les participants aux interactions sociales typifient leur comportement de manière à le rendre reconnaissable par les co-participants, ceux-ci évaluent en permanence le caractère reconnaissable et typifié du comportement auquel ils assistent. Ces formes typiques constituent autant de ressources interprétatives observables en contexte (point b) et soumises à une évaluation constante (point c).

b) Le caractère émergent et situé des pratiques. Les pratiques constituent avant tout des actions réalisées en contexte ; elles sont sociohistoriquement déterminées (un débat présidentiel en 1974 n'est pas strictement analogue à son pendant de 2007). Seule l'observation de récurrences parmi les pratiques effectivement réalisées peut fournir des

5. On est donc loin des formes d'expression politique usuellement étudiées en analyse des discours. Si l'on considère les travaux récents de la majorité des analystes du discours politique francophone (par ex. Mayaffre 2004 ; Burger 2005 ; Charaudeau 2005 ; De Chanay \& KerbratOrecchioni 2007 ; Adam 2008), force est d'avouer qu'un double critère préside à la sélection du corpus : de la politique française, sont privilégiées les interventions a) des élites du personnel politique b) produites dans un cadre médiatique professionnel.

6. Ainsi définie sur la quatrième de couverture de La parole polémique (2003).

7. Pour une présentation efficace, se référer à l'ouvrage de Filliettaz (2002: 42 et ss). 
informations sur les bases typifiées qu'admettent contractuellement les interactants pour s'orienter (point a).

c) Le caractère évalué des pratiques constitue le liant fondamental entre leur caractère typifié (abstrait, schématique) et leur caractère émergent $(\text { concret, réalisé })^{8}$. En d'autres termes, le fait que tel comportement produit en contexte soit reconnu comme telle action ayant telle finalité dépend de l'évaluation qu'en font les interactants qui y assistent. Du fait de l'unicité du contexte auquel il s'adapte, le comportement (par exemple l'éventuel comportement "polémique ») ne peut donc recevoir une lecture univoque, mais est évalué quant à sa typicalité : ressemble-t-il formellement à d'autres occurrences ayant eu une finalité analogue ?

d) L'importance donnée à la dimension langagière de l'interaction constitue un point central de l'interactionnisme socio-discursif. Le discours, espace de production de formes sémiotiques, constitue une ressource praxéologique centrale dans le dispositif d'évaluation et de reconnaissance des pratiques. Lieu d'intersubjectivité et d'intercompréhension, le discours en interaction constitue également le cadre d'apparition de diverses formulations permettant aux interactants d'expliciter et de négocier l'évaluation des conduites.

Établir l'existence d'une catégorie «polémique» dans une optique interactionniste implique donc de vérifier l'observabilité d'un pattern, que celui-ci soit discursif (le polémique) ou interactionnel (la polémique). L'existence de cette forme communicative assure alors l'analyste de son caractère évalué (en fonction de certaines finalités) et de sa disponibilité en contexte, les participants jugeant «assister à un discours polémique » ou «être en situation polémique», et accordant leur comportement en conséquence. L'observabilité du phénomène polémique repose ainsi sur le questionnement suivant : quels indices peut-on relever de l'orientation des participants vers l'existence d'une catégorie « polémique»?

\section{L'analyse du polémique en interaction : un bref état des lieux}

Par commodité, je synthétise ici trois recherches en analyse des discours et des interactions ayant le mérite d'avoir identifié des observables tendanciellement admis comme définitoires du ou de la polémique (KerbratOrecchioni 1980 ; Garand 1998 ; Amossy 2003). De tels observables constituent des ressources potentielles pour la description d'un «pattern

8. «Ce qui [...] me semble caractéristique de la "mouvance" interactionniste dans son ensemble, c'est le réinvestissement de la notion d'évaluation dans le cadre d'un rapport dialectique fondamental entre ce qu'on peut appeler les ressources typifiantes de l'agir et ses réalisations effectives » (Filliettaz $2002: 47$ ). 
interactionnel ou discursif» de la polémique, c'est-à-dire d'une forme communicative définie, typifiée et disponible pour les participants :

Verbalité et intertextualité : quand bien même le polémique peut se manifester de manière pluri-sémiotique, l'utilisation de ressources verbales est nécessaire. Il est en outre admis que l'entreprise polémique mobilise toujours un autre discours (par citation, par référence, par allusion) et se positionne vis-à-vis de lui.

Incorporation et nomination : le polémique engage fortement les locuteurs en tant qu'ils sont d'abord des êtres du monde. Il mise sur l'incarnation de la parole et donne ainsi une grande importance aux procédés de nomination. En vertu de la première régularité, les personnes restent néanmoins mobilisées en tant qu'elles tiennent ou ont tenu un discours.

Polarisation, disqualification et conflictualité : en situation polémique, les différends sont accentués et polarisés en positions antagonistes. Ceci s'accompagne de procédés de disqualification violente des personnes incarnant les positions.

Public et spectacularisation : le polémique se définit également par son caractère "constaté ». Il est réalisé face à un public-témoin qui emphatise la dimension spectaculaire de l'affrontement. Aujourd'hui, cette spectacularisation est largement affaire de médiatisation.

On doit admettre que le faisceau de critères ci-dessus est problématique par sa sous-détermination. Non seulement aucun critère n'est suffisant, mais tous sont tendanciels plutôt que strictement définitoires. On peut convenir néanmoins que la polémicité implique qu'un discours soit pris comme cible par un autre, et ce grâce à divers procédés de disqualification visant à décrédibiliser l'adversaire aux yeux d'un tiers. Les débats publics ayant été définis comme des dispositifs de communication favorisant l'incarnation des points de vue, on peut poser que ce genre de pratiques y est largement exploité.

Dans un premier temps, j'examine quelques extraits d'un débat public ${ }^{9}$ où l'engagement des personnes constitue un moyen d'attaquer les discours adverses. Ceci permet de considérer la forme discursive du polémique (4.).

9. Les extraits sont tirés d'un débat public ayant eu lieu le 4 octobre 2007 à l'Université de Lausanne et organisé par la Fédération d'associations d'étudiants de l'Université de Lausanne et le quotidien helvétique 24heures. Il réunit un public d'environ quatre-vingts personnes, un journaliste-animateur (ANIM), ainsi que dix candidats aux élections nationales (un candidat par parti politique) autour de la question de la relation entre études et emploi. Outre l'animateur, les extraits choisis font apparaître trois intervenants : Ada Marra (MARR) du Parti Socialiste, Julien Sansonnens (SANS) du Parti Ouvrier Populaire et Éric Bonjour (BONJ) de l'Union Démocratique du Centre. Les conventions de transcription sont consultables en annexe. 
Dans un second temps, je reprends l'ensemble des extraits d'un point de vue plus radicalement interactionnel, pour considérer la manière dont la phase évaluative peut soit confirmer simplement la présence d'un discours polémique (présence «du» polémique), soit entrainer la présence d'une interaction polémique (présence « de la » polémique) (5.).

\section{Le polémique dans le débat public}

Conformément aux observables traditionnellement dégagés, j'analyse le polémique comme un dispositif discursif articulant l'identification (le « ciblage ») d'un adversaire (4.1) et sa disqualification et/ou décrédibilisation face à un tiers (4.2).

\subsection{Cibler l'adversaire}

\section{EXTRAIT 1}

$\begin{array}{lll}1 & \text { MARR } & {[. . .] \text { alors on s'extasie face à ces centres de compétences mais ça }} \\ 2 & & \text { n'amène qu'un raisonnement de concurrencel (..) comme si seule la concurrence } \\ 3 & & \text { pouvait stimuler les esprits (.) heu moi je relève } \\ 4 & & \text { d'ailleurs\#1 les profs d'économie de Lausanne\#2 vont être contents }\end{array}$
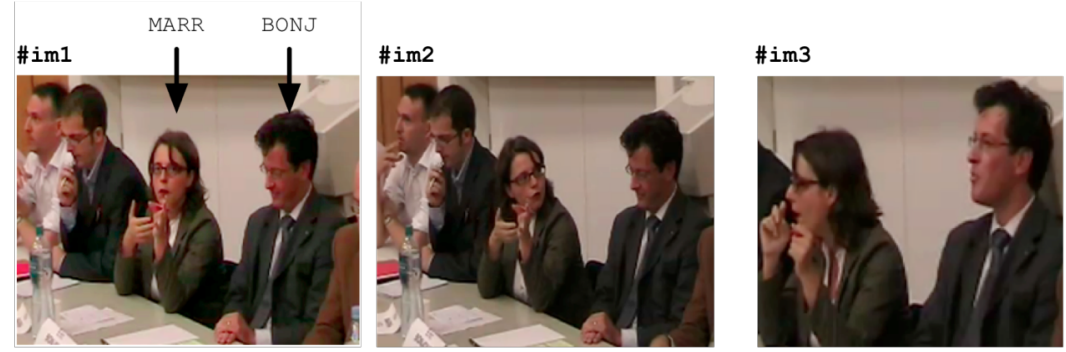

5

6 BONJ

7 MARR

8 BONJ

9 MARR

10 BONJ

11 MARR des propos de monsieur\#3 [heu de monsieur bonjour (...) y a un] peu\& [mais je (.) mais=j'CHUIS LIBRE DE MES PAROLESI]

\&les ignorants ici et les meil[leurs à HEC ${ }^{10}$ ] ben=voilà à SAINT GALL\&

[non=non c'est pas ce que j'dis]

\&c'est typique[ment:] ce genre de raisonnement qui faudrait éviter\& [pas ce que j'dis]

\&pour un regard critique (...) c'est c'que j'voulais direl

10. La locutrice fait référence aux propos tenus par BONJ quelques minutes auparavant. Défendant les pôles de compétence, BONJ avait valorisé les Hautes Études Commerciales (HEC) de Saint-Gall au détriment des institutions romandes, distinction reformulée par MARR en « les ignorants ici et les meilleurs à HEC [de Saint-Gall]». 
Dans cet extrait, MARR produit une incise au sein de son tour de parole (1. 3 et ss.), marquée tant verbalement ( «je relève d'ailleurs») que gestuellement (im1) et directionnellement (im2) ${ }^{11}$. Le ciblage mimo-gestuel de BONJ est néanmoins abandonné au moment même où celui-ci réplique en chevauchement (im3). Intervenant avant sa nomination par MARR (5-6), BONJ montre de son côté que le ciblage dont il fait l'objet ne souffre d'aucune ambiguïté ${ }^{12}$. MARR joue ainsi habilement avec la possibilité de diriger son énonciation contre BONJ par une conduite multimodale (mimogestuelle) tout en continuant à s'adresser au public et à refuser (par l'utilisation délocutive de la troisième personne) toute réciprocité conversationnelle avec l'adversaire ${ }^{13}$.

\section{EXTRAIT 2}

1 BONJ je veux dire cette réflexion elle vient du fait que les budgets

2 peuvent pas être augmentés ad aeternum on le voitl autrement on

3 aurait pas ce genre de discussionl=

4 DUMO =c'est un autre type [d'investissementl

5 BONJ [on serait tous d'accord pour les bourses\ (.)

$6 \quad[X X X X$

7 SANS [ben avec\#1 les cadeaux fiscaux que vous accordez c'est sûr que ça

8 va pas être augmenté\#2 de si tôtl
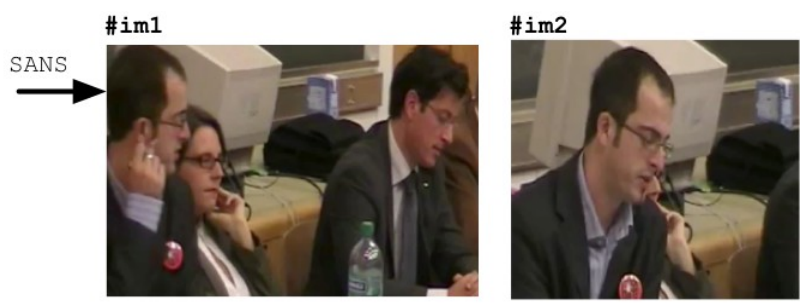

9 PUBL ((exclamations + rires))

11. Parmi les éléments para-verbaux, je distingue les gestes effectués par ostension et les postures directionnelles marquées par l'orientation du corps et du regard.

12. La mention "profs de Lausanne », par l'écho intertextuel qu'elle entretient avec la prise de position de BONJ quelques minutes auparavant (cf. note 11), participe certainement déjà à la désambiguïsation du ciblage. Quant à l'utilisation de la seconde personne, elle est plus complexe qu'elle n'y paraît, pouvant relever soit d'un "vous" de politesse (le locuteur s'adresse à son adversaire uniquement), soit d'un " vous " pluriel, incluant l'adversaire dans un groupe d'intérêt que la locutrice prend à parti (l'équivalent d'un « vous et ceux qui êtes avec vous »), dynamique fréquente dans les discours politiques et partisans.

13. La spectacularisation inhérente à la polémique trouve ici un écho explicatif: l'orateur (MARR dans le cas présent) cible et discrédite un adversaire (BONJ), mais ne perd pas de vue que le tiers, le public médiatique, reste l'arbitre de l'échange. 
Si le participant du deuxième extrait exploite également le ciblage directionnel (im1), l'intervention disqualifiante ne constitue pas ici une incise au sein d'un tour de parole. Au contraire, le tour de parole est, à proprement parler, l'occasion de l'intervention dépréciative, adressée cette fois directement à l'adversaire par l'usage de la seconde personne ${ }^{14}$. Notons néanmoins qu'au fil de son tour, SANS va détacher son regard de BONJ au profit d'une direction plus indéterminée, face au public (im2).

Dans un cas comme dans l'autre, le caractère incarné des points de vue et le jeu référentiel qu'il permet dans le choix du mode de désignation favorisent les deux dynamiques intrinsèques à la polémique : la sélection d'une cible et la spectacularisation. Dans l'opération polémique, la prise à partie d'un adversaire (en s'adressant directement ou non à lui) ne serait finalement qu'instrumentalisée au profit d'une mise en spectacle, faisant du public médiatique le témoin d'une attaque.

\subsection{Disqualifier et/ou décrédibiliser l'adversaire}

L'engagement des personnes à travers leur discours n'a pas manqué de susciter l'intérêt des spécialistes de la rhétorique et de l'argumentation. Plusieurs typologies ont été proposées pour distinguer et classer les différents procédés rhétoriques et schèmes argumentatifs favorisant la violence symbolique par la mise en cause des personnes au travers des discours. Deux grandes traditions typologiques se renvoient dos à $\operatorname{dos}^{15}$, mais les enjeux de l'opposition ne sont pas centraux ici. Les événements que j'analyse sont loin d'être avares en procédés de disqualification et de décrédibilisation de toute sorte, et ceux-ci peuvent sans peine être rangés sous l'étiquette générale d' " engagement conflictuel des personnes par les discours tenus ». Face à la diversité des pratiques, je me contenterai de relever quelques procédés en reprenant les deux extraits précédents et en y ajoutant les deux suivants :

14. À la différence du premier extrait, le locuteur s'exprime sans y avoir été autorisé (c.-à-d. sans avoir été hétérosélectionné) par l'animateur. Attaque sans médiation, cette intervention « du tac au tac » gagne peut-être en polémicité.

15. Les partisans d'une distinction radicale entre argumentations ad hominem et ad personam (voir entre autres: Arthur Schopenhauer, Gabriel Nucheldans, Chaïm Perelman et Marc Angenot) s'opposent aux partisans d'une conception intégrative de l'argument ad hominem (voir entre autres : Ruth Amossy et Douglas Walton). Ce différend terminologique est radical et complexe au point que l'ad hominem des seconds relève de l'ad personam des premiers. 


\section{EXTRAIT 3}

$\begin{array}{lll}1 & \text { BONJ } & \text { ouais faut plus de matières du- plus d'enseignement en matière dure/ } \\ 2 & & \text { mais pas forcément (.) les sciences heu di- dites annexesl (..) je } \\ 3 & & \text { vois que ça prête à sourire à certains mais je pense que c'est je } \\ 4 & & \text { pense qu'il faudra repasser par là/ et là c'est une orientationl } \\ 5 & & \text { politiquel (.) à reprendre et pis éviter cette génération soixante- } \\ 6 & & \text { huitardel (h) qu'on a [qu'on a vécu] jusqu'à maintenantl } \\ 7 & \text { PUBL } & \\ 8 & & \text { [OU::::::::::] } \\ 9 & \text { BONJ } & \text { voilàl }\end{array}$

\section{EXTRAIT 4} vos\&

\# im1

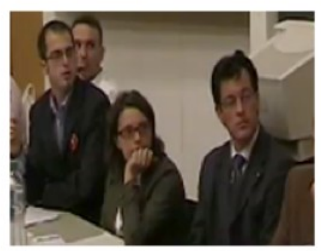

\# im2

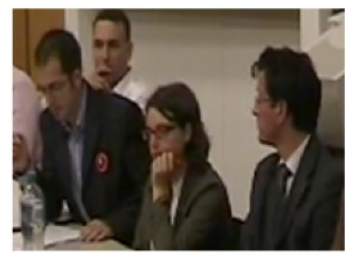

\# im3

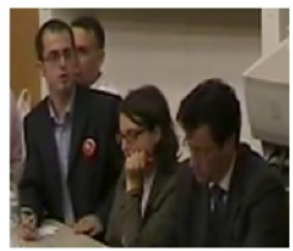

\&milieux\#3 (.) qui à chaque fois qu'il s'agit (.) d'engager UN centime d'argent public (.) dans dans une politique publique/ (.) nous expliquent qu'ils doivent être d'abord assignés au remboursement de la dette/ (.) ces mêmes milieux n'ont aucun état d'âme à faire reposer sur les épaules des étudiants et notamment les classes les plus modestes une dette pour rentrer dans le dans le début de la viel (.) c'est quand même pas normall

(..)

BONJ ben je m- je vous je vous retourne la question/ pourquoi dans d'autres cantons ça fonctionne et pas dans le canton de vaud/ c'est simplement une question de volontél il faut il faut se poser la question (..) p- une question de volonté/ c'est tout/ (1.0)

BONJ alors il faudrait poser la question ici ceux qui ont des bourses। (..) et ceux qui ont des prêts/ et pis il est clair que (.) déjà les études coûtent heu:: en ce qui concerne heu le cursus j'pense économique c'est quoi quinze mille francs par année le cursus médical (.) quarante mille francs par année/ (.) vous pouvez pas: non plus mettre de côté que vos études coûtent/ (.) donc il est clair/ comme disait monsieur heu bachmann\ (.) heu:.:. c'est [pas (.) un puits] sans fondl donc il est clair que nous on veut\& [vos millionnaires aussil] 


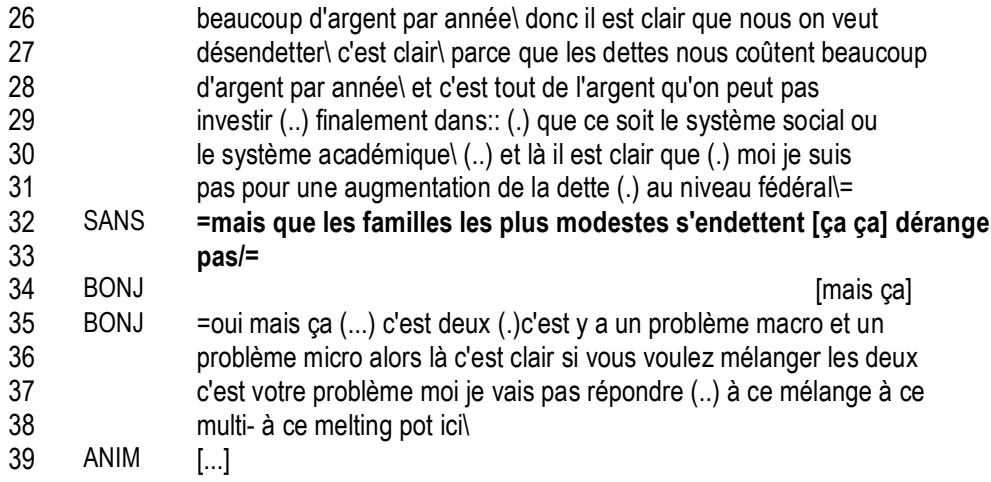

Dans l'extrait 1, la reformulation des propos de BONJ par MARR prend une valeur polémique par l'orientation négative qu'elle opère. La schématisation que propose MARR des propos de BONJ en tire les conséquences désobligeantes pour le public du débat («les ignorants ici»). Cette attaque ad hominem remet donc en cause la personne (ici sa moralité ou sa capacité de jugement) à travers la reprise orientée de ses propos.

Dans l'extrait 2, on pourrait gloser l'intervention de SANS de la manière suivante : "même si vous l'admettez unanime, vous êtes le moins bien placé pour défendre notre cause parce que vous agissez en sa défaveur ». Dans la typologie de Douglas Walton, cette attaque relève d'un Universal circumstantial Ad hominem (Walton 1998 : 253 ; Walton et al. 2008 : 354). Il s'agit de décrédibiliser l'argumentation de la cible en argumentant que ses actes («vous réduisez les recettes fiscales de l'État») entrent en contradiction avec sa défense d'une thèse prétendue universelle ("tout le monde voudrait les bourses, mais c'est l'argent qui manque»). D'après Walton, cette mise en contradiction aboutit sur la disqualification morale de la cible (critique d'insincérité) et l'invalidation de sa proposition.

L'extrait 3 témoigne de la polémicité de certaines formules (voir par exemple Krieg-Planque 2009 : 116). Ainsi, " Mai soixante-huit » couplé à l'extensif «génération» et au suffixe «-ard» participe d'une stéréotypification dépréciative. Aucun participant présent n'est explicitement ciblé - d'un point de vue multimodal, BONJ s'adresse frontalement au public -, mais la critique entre en écho avec les prises de position antérieures de plusieurs adversaires.

Finalement, l'intervention de SANS (2-9) dans l'extrait 4 relève de ce que D. Walton appelle une Logical Inconsistency circumstantial ad 
hominem. Il s'agit de la variante ad hominem $^{16}$ de l'Inconsistent Commitment ("You contradict yourself») : l'adversaire est accusé («ce n'est pas normal ») de suivre deux logiques incompatibles entre elles et dont l'incompatibilité est en outre moralement condamnable (condamner l'endettement public, mais ne pas vouloir éviter l'endettement privé). L'attaque est complexe dans le sens où elle allie la disqualification de la moralité (absence d'empathie, opportunisme) et la décrédibilisation de la performance oratoire (contradiction dans l'attitude). Si BONJ écarte l'accusation de contradiction dans un premier temps (16-31), il s'en défend après la nouvelle sollicitation de SANS (32-33) et ceci en invoquant précisément la dissociation des notions (personnes VS institutions) et en condamnant le brouillage opéré par l'attaquant.

Ces extraits ont permis de considérer quelques actualisations du discours polémique. Il est possible d'en proposer une schématisation triviale :

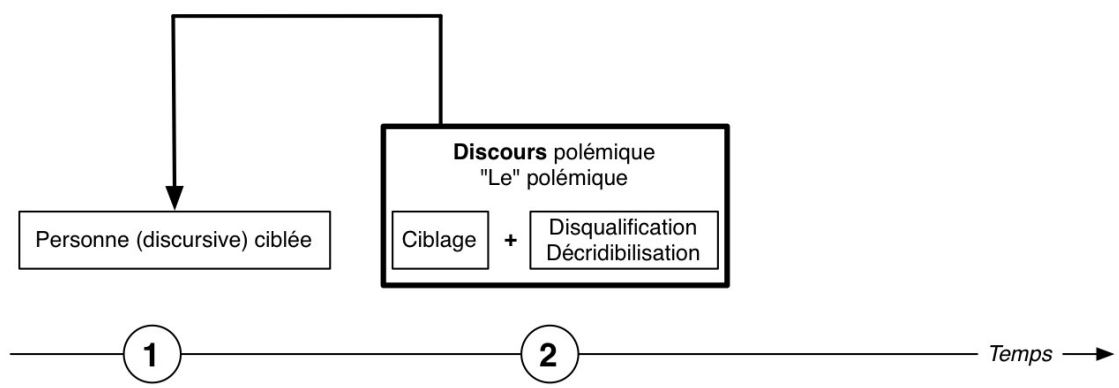

Figure 1 : La forme discursive du polémique prise dans l'interaction

Le polémique constitue une forme communicative disponible en situation argumentative. Elle consiste à argumenter par disqualification (attaque directe sur la personne, pouvant s'apparenter à l'insulte) et/ou décrédibilisation (mise en faux, mise en contradiction) de l'adversaire face à un tiers. Cette définition traditionnelle du polémique s'est vérifiée dans les données. Elle pose cependant au moins deux problèmes complémentaires :

(i) La définition omet le fait que "polémique» n'est pas un terme neutre et donc qu' « en désignant un échange par le terme polémique, le locuteur prend position sur cet échange » (Plantin 2003 : 401). Le constat est aussi valable pour l'analyste : qualifier une conduite de "polémique ",

16. Dans la typologie de Douglas Walton, relève de l'ad hominem tout raisonnement argumentatif passant par une étape de disqualification explicite de l'adversaire ("bad person » en 1998, "person of bad character » en 2008), que cette étape soit un posé ou une conclusion intermédiaire pour la sanction de l'argumentation ciblée. 
c'est immédiatement l'évaluer de manière axiologique. Comment transformer en avantage - voire en appui méthodologique - le danger que constitue pour l'analyste l'usage du terme « polémique »?

(ii) La définition, si elle nous permet de saisir les «traits caractéristiques » du polémique, ne nous permet pas encore de comprendre la polémique. En quoi change-t-on de régime ou de niveau lorsqu'on passe d'une action verbale évaluée comme polémique à un échange interactionnel polémique ? Ainsi, dans une situation intrinsèquement agonale comme le débat (Plantin $2003 \& 2005$; Burger 2005), quand s'arrête ce dernier et où commence la polémique?

\section{Jugement de polémicité et interaction polémique}

Les extraits de mon corpus ne se limitent pas à rendre manifestes des formes traditionnellement rangées dans le polémique. De fait, ils font plus que témoigner d'un engagement disqualifiant et/ou décrédibilisant d'un adversaire face à un tiers, car ils montrent aussi une résistance : l'adversaire devenu la cible d'une attaque - il peut également s'agir d'un tiers prenant sa défense - ne se laisse pas faire. Il intervient sans passer par la médiation de l'animateur, il hue, il proteste, il condamne. En d'autres termes, il sanctionne le mouvement argumentatif de l'attaquant, il rend manifeste l'évaluation qu'il fait de la conduite à laquelle il assiste. L'analyste n'a en ce sens pas à «prendre position», pour reprendre les termes de Plantin. Il lui suffit de recueillir les traces d'une orientation des participants vers le caractère «déjà » évalué d'une forme, en l'occurrence les traces d'un jugement de polémicité.

Ainsi, dans l'extrait 1, BONJ s'auto-sélectionne et conteste l'attaque de MARR en chevauchant son tour de parole ( je suis libre de mes paroles »; « ce n'est pas ce que j'ai dit»; «pas ce que j'ai dit»). L'évaluation qu'il produit des propos de MARR prend ainsi la forme d'une sanction de polémicité. La protestation de BONJ étant ignorée par MARR qui se tourne vers le public, cette situation conflictuelle ne donne pourtant pas lieu à un véritable échange.

Dans l'extrait 2, l'intervention de SANS donne lieu à des rires et à des protestations. Ces jugements (valorisation pour les rires, sanction pour les protestations) viennent confirmer le caractère spectaculaire du polémique. La dynamique est similaire dans l'extrait 3 , où la huée d'un membre du public vient sanctionner le caractère polémique de la formule "génération soixante-huitarde ». Dans les deux cas, le public médiatique - assumant son rôle de juge (Plantin 2005) - évalue l'agression dont il est le témoin.

Finalement, dans l'extrait 4 , BONJ achève son tour en exprimant un refus de répondre à la réitération de la question de SANS. Ce refus, doublé 
par la condamnation du brouillage des pistes opéré par l'adversaire, relève d'une sanction de polémicité tentée par l'orateur pour se défendre de l'attaque de l'adversaire.

Ainsi, si les termes de «polémique» ou de "polémiquer» n'apparaissent pas dans les extraits sélectionnés ni d'ailleurs dans le reste $\mathrm{du}$ corpus, l'orientation discursive et interactionnelle des locuteurs vers la production de divers jugements de polémicité garantit la présence d'une forme communicationnelle. Ainsi, ce qu'on a pu identifier comme action polémique, définie par la présence d'une attaque (disqualification et/ou décrédibilisation) produite, face à un tiers, par un débattant à l'encontre d'un autre, ne passe pas inaperçu. Que la réaction soit (i) celle de la cible de l'attaque ou d'un témoin prenant sa défense (sanction), ou (ii) celle d'un tiers appréciant l'aspect spectaculaire de l'agression verbale (valorisation), la polémicité d'un mouvement argumentatif est affaire d'évaluation située et rendue manifeste.

Le jugement de polémicité peut alors donner lieu à une polémique, un échange interactionnel basé sur une alternance d'actions discursives polémiques et de sanctions réciproques - où chacun accuse l'autre d'enfreindre les règles de l'argumentation et du débat et par conséquent de polémiquer, comme le montre la figure 2 ci-dessous :

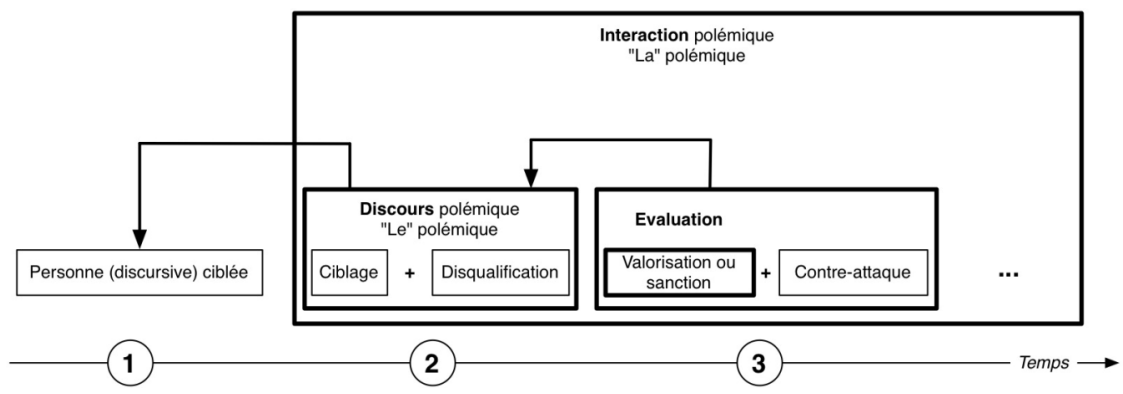

Figure 2 : La forme interactionnelle de la polémique

Dans les extraits 1-3, la cible (ou la personne qui prend sa défense) se contente de sanctionner l'action polémique. L'évaluation ne s'accompagne pas pour autant d'une contre-attaque aboutissant à une véritable alternance "polémique » des locuteurs. L'extrait 4 contraste, ne serait-ce que par la durée du moment polémique dont il témoigne : SANS et BONJ s'attaquent mutuellement quant aux motivations qu'ils s'imputent et à l'esprit dont témoignent leurs actes ou ceux de leur parti, le tout aboutissant, suite à la réitération par SANS de son attaque initiale, à la sanction de polémicité exprimée par BONJ et que nous avons déjà commentée. Si l'échange 
s'oriente vers la polémique du fait de la réciprocité des attaques produites, la dynamique interactionnelle ne tourne cependant pas à la sanction réciproque de polémicité.

\section{Conclusion}

À notre question de départ, interrogeant l'opportunité d'utiliser la catégorie polémique pour l'analyse de débats publics, nous pouvons maintenant répondre par l'affirmative. Effectivement, il y a lieu d'identifier « du » polémique (c'est-à-dire une action discursive) et « de la » polémique (c'est-à-dire un type d'échange interactionnel). Mais cette identification, du fait $\mathrm{du}$ caractère fondamentalement axiologique du terme même de «polémique», doit être méthodologiquement instrumentée. En effet, la manipulation prudente qu'exige la catégorie « polémique » n'en exclut pas pour autant toute scientificité. Bien au contraire. Il s'agit - et l'enjeu est crucial - de penser la charge axiologique de la catégorie relativement au comportement même des agents sociaux. Autrement dit, au lieu de laisser à l'analyste la charge de l'évaluation, il s'agit de relever les indices que ceuxci laissent de leur orientation vers l'accomplissement du ou de la polémique, l'idée étant que les participants aux interactions évaluent certaines conduites comme relevant du polémique et manifestent eux-mêmes un «être-ensituation-polémique ». Le jugement de polémicité prend avant tout la forme d'une sanction : celui qui juge faire les frais d'une action polémique (ou celui qui prend la défense de la cible de l'agression) s'en indigne et conteste sa validité ou son opportunité ${ }^{17}$. Il se peut toutefois que le jugement de polémicité prenne la forme d'une valorisation : dans ce cas, c'est la logique spectaculaire - emphatisée par la dimension médiatique des événements du fait polémique qui l'emporte, un tiers manifestant un certain amusement face à l'infraction ${ }^{18}$. Ainsi l'argumentation tourne au polémique et le débat à la polémique lorsque des conduites sont sanctionnées en vertu des normes en vigueur.

Parmi les raisons pour lesquelles un comportement argumentatif d'un locuteur peut être sanctionné par un autre, nous avons vu que les traits

17. Cela ne signifie pas pour autant que les actions incriminées tout autant que leurs évaluations ne soient pas attendues. Tout est affaire de genre d'interaction : un débat politique médiatisé (même minimalement comme dans le cas des débats publics) implique une certaine exacerbation de la conflictualité, situation garantissant le caractère spectaculaire de l'interaction proposée au public médiatique.

18. Nous ne saurions trop souligner ce point: si la polémique est affaire d'infraction argumentative, ce n'est pas pour autant que le cadrage médiatique et sa logique de spectacularisation l'interdisent, bien au contraire. Être sanctionné par un adversaire pour son action polémique n'implique pas de l'être également par le public, destinataire «final» du propos. 
définitoires traditionnellement avancés dans l'étude du fait polémique se vérifient : dans les extraits considérés, les énoncés sanctionnés possèdent tous les marques d'un engagement disqualifiant et relativement spectaculaire des personnes. Cet engagement peut relever de l'insulte (extrait 3), de l'attaque de moralité (extrait 2), de la reformulation de propos tenus (extrait 1) ou encore de la mise en contradiction (extrait 4). Que la personne soit attaquée directement, que l'attaque personnelle serve à discréditer la thèse ou encore que discréditer la thèse permette de sanctionner la personne qui la soutient, le point commun de ces stratégies, source des jugements de polémicité produits par les acteurs, consiste en une gestion conflictuelle des faces, disqualifications à l'appui. Si les tentatives de disqualification et/ou de décrédibilisation de l'autre sont traitées comme des infractions polémiques, on ne saurait prêter aux normes qu'elles enfreignent une valeur universelle. Ce que les participants traitent comme infraction argumentative ne fait que refléter - en miroir inversé - le caractère socio-historiquement déterminé des normes qu'ils se donnent pour gérer leurs interactions. Ainsi, si les mouvements argumentatifs se fondant sur la relation argumentspersonnes sont jugés polémiques, on peut faire l'hypothèse que les jugements de polémicité se fondent sur l'idéal occidental de rationalité, dans lequel les raisons doivent trouver des fondements objectifs, hors de la prise en considération des personnes (Angenot 2008).

Si les analyses classiques de la polémique témoignent selon Plantin (2003) d'une conception naïve et angélique de l'argumentation (qu'elles opposent au polémique) et du débat (qu'elles opposent à la polémique), elles n'en restent pas moins empiriquement fondées : le comportement des acteurs réfléchit la définition de la polémique qu'ils se donnent et à l'aune de laquelle ils évaluent leur participation au dialogue politique ${ }^{19}$.

\section{Références bibliographiques}

ADAM, Jean-Michel (2008 [2005]), La linguistique textuelle: introduction à l'analyse textuelle des discours, Paris, Armand Colin.

Amossy, Ruth (2003), "L'argument Ad hominem dans l'échange polémique », dans Gilles De ClerCQ, Michel Murat et Jacqueline DANGel (éds), La parole polémique, Paris, Honoré Champion, 409-423.

Angenot, Marc (2008), Dialogues de sourds: traité de rhétorique anti-logique, Paris, Mille et une nuits.

BroncKART, Jean.-Paul (1997), Activité langagière, textes et discours : pour un interactionnisme socio-discursif, Lausanne ; Paris, Delachaux et Niestlé.

19. L'auteur tient à remercier Ruth Amossy, Marcel Burger et Raphaël Micheli pour leur relecture attentive et leurs précieux conseils. Il garde néanmoins la responsabilité de toutes les maladresses et imprécisions qui subsisteraient. 
BuRger, Marcel (2005), «La complexité argumentative d'une séquence de débat politique médiatique», dans Marcel BURGER et Guylaine MARTEL (éds), Argumentation et communication dans les médias, Québec, Nota Bene.

Charaudeau, Patrick (2005), Le discours politique : les masques du pouvoir, Paris, Vuibert.

Constantin De Chanay, Hugues et Kerbrat-Orecchioni, Catherine (2007), « 100 minutes pour convaincre : l'éthos en action de Nicolas Sarkozy », dans Mathias Broth et al. (éds), Le français parlé des médias, Stockholm, Acta Universitatis Stokholmiensis, 309-329.

FilliettAZ, Laurent (2002), La parole en action. Éléments de pragmatique psychosociale, Québec, Nota Bene.

GARAND, Dominique (1998), «Propositions méthodologiques pour l'étude du polémique », dans Annette HAYWARD et Dominique GARAND (éds), États $d u$ polémique, Québec, Nota Bene, 211-268.

HutchBy, Ian (1996), Confrontation Talk: arguments, asymmetries, and power on talk radio, Mahwah, N.J., L. Erlbaum Associates.

Kerbrat-OrecCHIONI, Catherine (1980), « La polémique et ses définitions », dans Nadine GELAS \& Catherine KERBRAT-ORECCHIONI (éds), Le discours polémique, Lyon, PUL, 3-40.

KRIEG-PlanQue, Alice (2009), La notion de formule en analyse du discours : cadre théorique et méthodologique, Besançon, PUF-C.

MAYAFFre, Damon (2004), Paroles de président. Jacques Chirac (1995-2003) et le discours présidentiel sous la Vème République, Paris, Champion.

Plantin, Christian (2003), «Des polémistes aux polémiqueurs », dans Gilles DE ClercQ, Michel Murat et Jacqueline DANGel (éds), La parole polémique, Paris, Honoré Champion, 377-408.

Plantin, Christian (2005), L'argumentation : histoire, théorie et perspectives, Paris, PUF.

Walton, Douglas (1998), Ad Hominem Arguments, Tuscaloosa, University of Alabama Press.

Walton, Douglas, Reed, Christopher et Macagno, Fabrizio (2008), Argumentation Schemes, Cambridge, CuP.

Z'GRAGgen, Heidi et Linder, Wolf (2004), Professionalisierung der Parlamente im internationalen Vergleich, Berne, étude sur mandat des services du Parlement de l'Assemblée fédérale.

\section{Conventions de transcription}

$<(($ rit $))$ non $>$

(.) $(.).($...)

(1.5)

FAMEUX

Fameu::se

$\mathrm{X}$ XX

$\&$

[] commentaire : para-verbal ou non-verbal, situationnel, etc. micropauses : fractions d'une seconde pause en secondes volume saillant allongement mot inaudible, séquence inaudible ( $1 \mathrm{X}=1$ syllabe $)$ prolongement du tour à la ligne suivante chevauchement 


$\begin{array}{ll}1 & \text { intonation montante } \\ \text { \#N } & \text { intonation descendante } \\ \text { image capturée de la vidéo }(\mathrm{N}=\text { numéro de référence) }\end{array}$

\title{
Le/La polémique : une catégorie opératoire pour une analyse discursive et interactionnelle des débats publics ?
}

Résumé : Cette contribution questionne le caractère opératoire de la catégorie «polémique» pour l'analyse de débats publics. Après avoir présenté les traits traditionnellement avancés pour définir la catégorie, deux questions sont abordées : (i) le caractère polémique d'une forme communicationnelle semble dépendre de l'évaluation produite en contexte par les agents sociaux; (ii) il semble judicieux d'opérer une distinction entre forme discursive "polémique» et type d'activité interactionnelle "polémique». La contribution se termine par une réflexion sur l'importance de considérer l'évaluation comme catégorie sociohistorique: si les participants jugent passer de l'argumentatif au polémique et du débat à la polémique, ils le font à l'aune de normes socialement et historiquement déterminées distinguant le licite et l'illicite.

Mots-clefs : Le polémique, La polémique, Évaluation, Discours, Interaction, Débats publics

\section{Is controversy a profitable category for a discursive analysis of public debates?}

\begin{abstract}
This article aims at evaluating the profitability of the category "polemical" for the analysis of public debates. After giving a traditional definition of the category, two issues are tackled: (i) the polemical nature of a form of communication seems to depend on the evaluation that the participants produce in context; (ii) it seems that it would be wise to distinguish between the discursive form, i.e., "polemic", and the interactional activity, which is "polemical". The paper ends on a discussion about the importance of considering evaluation as a socio-historically determined process: when participants evaluate a talk or an interaction as "polemical", they do so on the basis of sociologically and historically determined norms that distinguish licit from illicit argumentative moves.
\end{abstract}

Keywords: Polemical talk, Polemical interaction, Evaluation, Public debates 